\title{
Norois
}

Environnement, aménagement, société

$232 \mid 2014$

Modes de vie, modes d'habiter des aînés entre inclusion et exclusion

\section{Politiques visant la santé au Québec : lorsque la main droite ignore ce que fait la main gauche}

Health policies in Quebec: when the right hand doesn't know what the left hand is doing

Alexandre Couture Gagnon et Jean-François Savard

\section{OpenEdition}

Journals

\section{Édition électronique}

URL : https://journals.openedition.org/norois/5183

DOI : $10.4000 /$ norois.5183

ISSN : $1760-8546$

\section{Éditeur}

Presses universitaires de Rennes

\section{Édition imprimée}

Date de publication : 30 octobre 2014

Pagination : 81-87

ISBN : 978-2-7535-3974-7

ISSN : 0029-182X

\section{Référence électronique}

Alexandre Couture Gagnon et Jean-François Savard, «Politiques visant la santé au Québec : Iorsque la main droite ignore ce que fait la main gauche », Norois [En ligne], 232 | 2014, mis en ligne le 30 octobre 2016, consulté le 14 janvier 2022. URL : http://journals.openedition.org/norois/5183 ; DOI : https:// doi.org/10.4000/norois.5183 


Presses
Universilaires
wwe Rentes

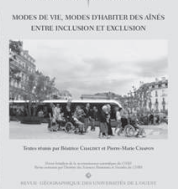

Note de recherche

\title{
Politiques visant la santé au Québec : lorsque la main droite ignore ce que fait la main gauche
}

\author{
Health Policies in Quebec: When the Right Hand doesn't Know what the Left Hand is Doing
}

\author{
Alexandre Couture Gagnon ${ }^{* a}$, Jean-François Savard ${ }^{b}$
}

\footnotetext{
* Auteur correspondant

a Assistant professor, University of Texas Rio Grande Valley, One West University Boulevard, BrownsviLle, Texas

78520, USA (alexandre.couturegagnon@utb.edu)

b Professeur, École nationale d'administration publique, Pavillon Alexandre-Taché, aile D - 283, boulevard

Alexandre-Taché, C.P. 1250, Gatineau J8X 3X7, Québec, Canada (Jean-Francois.Savard@enap.ca)
}

Résumé : Cet article analyse la cohérence des politiques publiques de santé et de transport au Québec. Il conclut que, tandis que la formulation de ces politiques est cohérente, leur mise en œuvre s'avère incongruente. Ainsi, la politique de santé du Québec vise notamment à réduire les décès et maladies dus à la pollution de l'air, en ligne avec la politique de transport du Québec, laquelle souhaite réduire les émissions des gaz à effet de serre. Or, la mise en œuvre de ces politiques ne permet pas d'atteindre ces objectifs en raison de la densité de la hiérarchie, c'est-à-dire le nombre de hiérarchies (Hupe, 2010). Autrement dit, la décentralisation des deux ministères concourt à une mauvaise interprétation des priorités conjointes des ministères.

Abstract: This article analyzes the coherence of public health and transportation policy in Quebec. It concludes that, while the formulation of these policies is coherent, their implementation is incongruent. Health policy in Quebec aims, among other things, at lowering deaths and diseases caused by air pollution; and transportation policy in Quebec aims at lowering greenhouse gas emissions. Yet the implementation of these policies does not make it possible to reach this target because of thickness of hierarchies, that is, the number of hierarchies (Hupe, 2010). Otherwise said, decentralization in the two departments leads to wrong interpretation of joint priorities.

Mots clés : Politiques publiques - cohérence - santé, transport - Québec - décentralisation

Keywords: Public policy - coherence - healthcare - transportation-Quebec-decentralization 


\section{INTRODUCTION}

Au Québec, les objectifs du Programme national de santé publique (Québec, 2008c) et du Plan d'action gouvernemental de promotion des saines habitudes de vie et de prévention des problèmes reliés au poids (Québec, 2006a) visent la réduction d'une série de maladies liées à des facteurs qui caractérisent l'environnement des citoyens, notamment par l'adoption d'approches touchant la réduction des gaz à effet de serre, l'aménagement urbain, le transport durable et la promotion d'un mode de vie actif et d'une saine alimentation. Parallèlement, les objectifs du Plan stratégique 2008-2012 (Québec, 2008a) du ministère des Transports, ainsi que la Politique québécoise du transport collectif (Québec, 2006b) et la Politique sur le vélo (Québec, 2008b) visent à soutenir des systèmes de transport contribuant à la réduction des émissions de gaz à effet de serre, à accroître l'utilisation du transport collectif et à encourager l'usage du vélo et le développement de voies cyclables.

L'analyse des politiques de ces deux domaines nous permet de constater que si le langage des politiques est différent, les effets recherchés se complètent. On peut affirmer qu'il existe une harmonie des objectifs entre ces politiques. En effet, en développant un système de transport qui contribue à la réduction des gaz à effet de serre, le Plan stratégique (Québec, 2008a) du ministère des Transports vient renforcer le Programme national de santé publique (Québec, 2008c) qui cherche à réduire les maladies liées aux émissions de gaz à effet de serre et autres polluants. Aussi, les politiques de santé visent à favoriser un mode de vie actif et une saine alimentation, des objectifs qui sont renforcés par ceux des politiques sur le transport en commun et le vélo, qui encouragent non seulement un mode de vie actif, mais contribuent également à agir positivement sur des facteurs de risque liés à plusieurs maladies chroniques en diminuant la quantité de polluants émis dans l'air.

Bien qu'il existe une certaine harmonie des objectifs de ces politiques, l'analyse de la mise en œuvre de ces politiques révèle une différence significative entre les objectifs énoncés et leur implémentation. Par exemple, alors que la politique de transport prévoit le développement de modes de transport plus efficaces pour réduire les émissions de gaz à effet de serre, dans les faits, le ministère des Transports cherche à mettre en place un système de modalité de transport plus efficace et non pas à améliorer la qualité de l'air en tant que telle (Québec, 2013c). De même, en santé, malgré la volonté du ministère de réduire les facteurs de risque environnementaux, peu d'actions semblent avoir été posées en ce sens (Québec, 2014a). Il apparaît évident que le ministère contrôle bien peu ces enjeux (Québec, 2014c). Il semble donc y avoir un décalage entre la formulation des objectifs de ces politiques et leur mise en œuvre.

\section{Mise en GUVRE incongruente}

Comment expliquer ce décalage? Selon Hupe (2010), il s'agit d'une mise en œuvre incongruente. Cette idée n'est pas nouvelle. Au contraire, Pressman et Wildavsky (1973) l'ont évoquée dès 1973. Dans leur ouvrage, ils soutenaient que les probabilités que des programmes fédéraux américains puissent être mis en œuvre dans les localités étaient infimes étant donné la lourdeur du processus de politiques publiques que cela impliquait. Ils affirmaient ainsi que plus une chaîne verticale de mise en œuvre comportait de pôles décisionnels (clearances), plus les chances d'une mise en œuvre incongruente étaient grandes (Hupe, 2010). Il faut souligner que cette thèse de Pressman et Wildavsky a été contestée par plusieurs auteurs, dont Bowen (1982), qui remettaient en question la validité des arguments. Malgré ces objections, nous croyons qu'il est possible d'observer une cohérence des politiques dans leur formulation, mais un degré élevé d'incohérence dans leur mise en œuvre, qui s'explique par la thèse de la mise en œuvre incongruente. Il y a incohérence de politiques publiques lorsque les objectifs ou les actions, analysés séparément, de deux ministères ou entités ont des conséquences différentes.

Nous retenons de Hupe l'idée que cette incongruence de la mise en œuvre s'explique par ce qu'il appelle la densité de la hiérarchie (thickness of hierarchies). Ainsi, selon cet auteur, la mise en œuvre des politiques publiques se déroule dans un contexte où un grand nombre de hiérarchies sont impliquées. Ce n'est donc pas seulement le nombre de pôles décisionnels qui influe sur la capacité de mettre en œuvre les politiques, mais également le nombre de hiérarchies. Par exemple, deux ministères auront leur hiérarchie respective et les décisions prises dans les hiérarchies de chacun de ces ministères auront des 


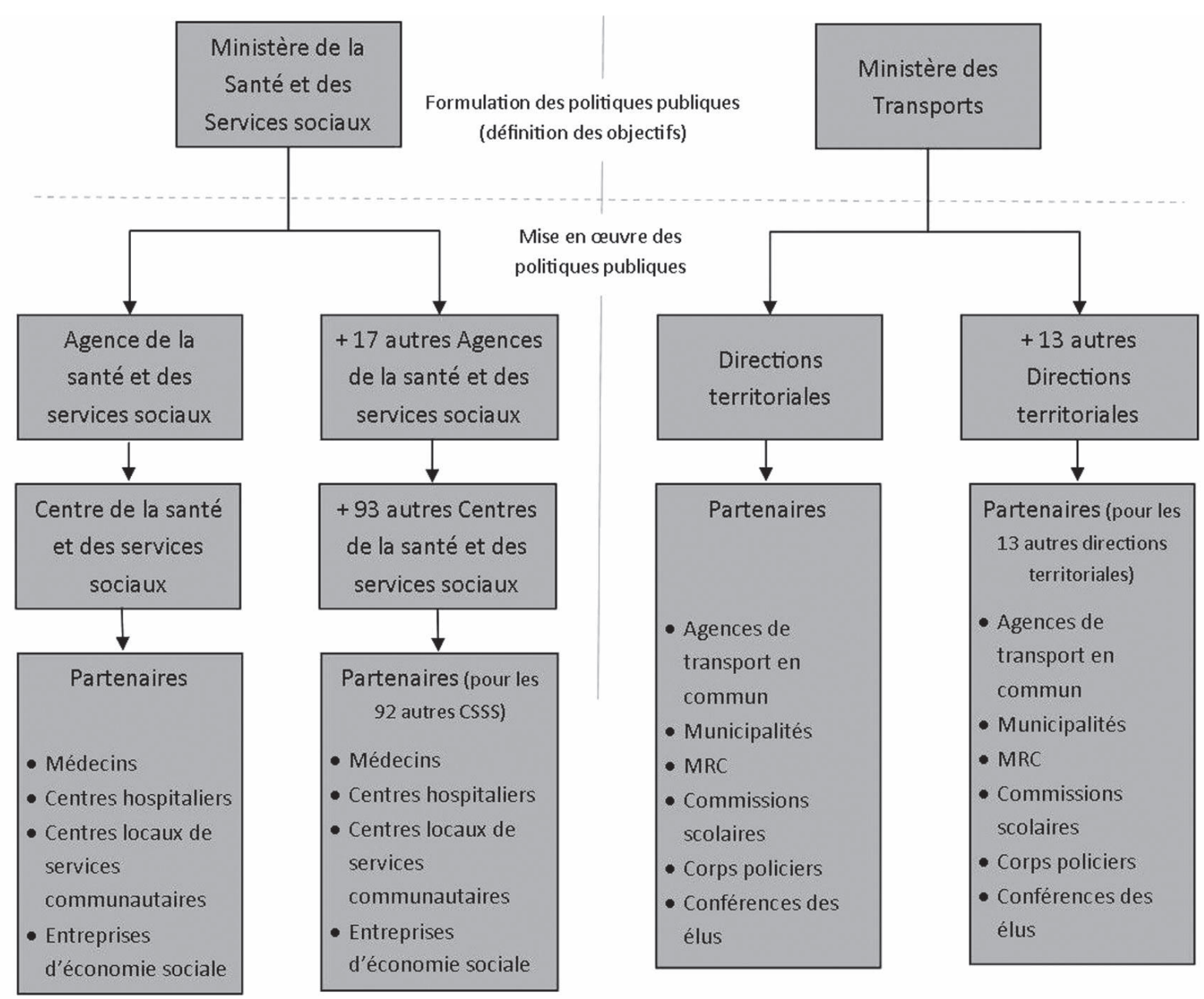

Figure 1 : Formulation et mise en œuvre des politiques aux ministères de la Santé et de Services sociaux du Québec et des Transports Formulation and implementation of policies in Healthcare and Social services Department and Transportation Department

effets sur la mise en œuvre des politiques. Plus il y a de hiérarchies différentes (on parle alors de nombre de hiérarchies), plus la densité est élevée. C'est pour cette raison que nous dirons que ces hiérarchies ne sont pas liées par des relations symétriques; mais qu'elles jouissent plutôt d'un certain degré d'autonomie dont le résultat est la création d'un univers de mise en œuvre laissant place à de nombreuses interprétations des objectifs et des exigences d'une politique. Conséquemment, plus une politique repose sur des objectifs normatifs ou des objectifs plus ou moins bien définis, plus la densité de la hiérarchie profite aux diverses interprétations autonomes de la politique et plus l'incongruence de la mise en œuvre est grande (Hupe, 2010).

\section{Méthodologie}

Notre recherche s'appuie sur une analyse documentaire classique. Nous avons d'abord recensé des sites Internet des ministères québécois de la Santé et des Services sociaux et des Transports, afin de trouver les documents ayant trait aux politiques de transport et de santé (énoncés de politiques, guides de mise en œuvre, rapports de suivi, discours des ministres) nous permettant de bien comprendre les liens entre les politiques de santé et de transport. Par la suite, nous avons procédé à une analyse de contenu de type thématique des documents que nous avions répertoriés. Pour ce faire, nous avons procédé par codage inductif des documents pour en extraire les objectifs des politiques de transport et de santé et les actions de mise en ouvre de 
ces politiques (qui sont parfois exprimés de façon explicite, mais le plus souvent de façon implicite). Au terme du codage, nous avons été en mesure de créer des grilles thématiques répertoriant l'ensemble des objectifs et des actions de mise en œuvre des politiques de transport et de santé. À l'aide de ces grilles, nous avons par la suite comparé les objectifs et les actions de mise en œuvre entre les politiques de transport et les politiques de santé de façon à déterminer à quels objectifs se rattachaient les actions de mise en œuvre entreprises dans le cadre des politiques de transport et de santé, et à observer s'il y avait des recoupements entre les objectifs et les actions de mise en œuvre de l'un et de l'autre de ces domaines. Nous avons pu ainsi apprécier la cohérence de la formulation de ces politiques et la cohérence de leur mise en œuvre.

\section{INCONGRUENCE DE LA MISE EN GUVRE AU QUÉBEC}

L'analyse de la structure de mise en œuvre des politiques de santé et de transport au Québec montre bien l'existence de chaînes verticales caractérisées par de nombreux pôles décisionnels et une grande densité des hiérarchies. En effet, au Québec, les agences de santé sont les entités responsables de la mise en œuvre des objectifs du ministère de la Santé et des Services sociaux du Québec, alors que le ministère des Transports du Québec s'en remet à ses directions territoriales. Le ministère de la Santé et des Services sociaux met en ouvre ses programmes par l'entremise de dix-huit régions qui se partagent la province (Québec, 2014b:6). En retour, les dix-huit agences sont « [responsables de] coordonner la mise en place des services sur leur territoire respectif » en fonction des budgets qui leur sont alloués (Québec, 2014b : 5). Enfin, ces agences se déploient dans un dernier palier de gouvernance, centré autour de quatre-vingt-quatorze centres de santé et de services sociaux (CSSS), qui regroupent les «partenaires » (médecins de famille, centre hospitalier, entreprises d'économie sociale, etc.) (Québec, 2014b : 9, 13).

À l'instar du ministère de la Santé et des Services sociaux, la mise en œuvre au ministère des Transports du Québec est décentralisée : ce dernier compte quatorze directions territoriales. Le ministère est responsable de « l'organisation des systèmes de transport au Québec » (Québec, 2013b). Toutefois, la mise en ouvre est du ressort des directions territoriales. Celles-ci décident, en collaboration avec les partenaires du milieu (autorités de transport en commun, municipalités, municipalités régionales de comté, commissions scolaires, corps policiers, conférences des élus, etc.) de l'allocation des ressources. Les directions territoriales rédigent les plans de transport pour leur territoire, décrits comme «l'outil de planification privilégié par le ministère des Transports pour guider ses actions futures» (Québec, 2013a).

Le ministère des Transports et le ministère de la Santé et des Services sociaux présentent des systèmes de décentralisation différents. Les directions territoriales des Transports ont un lien hiérarchique direct avec le ministère; les directeurs territoriaux se rapportent à leur sous-ministre adjoint et parfois vont même travailler avec le sous-ministre. À la Santé, les dix-huit agences de santé publique n'ont pas de lien hiérarchique direct avec le ministère. Elles reçoivent des directives du ministère, elles touchent leur budget du ministère, mais elles ont aussi une autonomie d'action et de décision. Elles ont à leur tête un conseil d'administration dont certains membres sont des élus issus de la population.

Ainsi, au Québec, les politiques de santé ne sont généralement pas mises en œuvre par le ministère, mais bien par les dix-huit agences de santé qui couvrent le territoire québécois. Aussi, la Loi sur la santé publique confère au ministère de la Santé et des Services sociaux un rôle de premier conseiller en matière de santé et oblige tous les ministères et organismes gouvernementaux à le consulter si leurs lois peuvent avoir un impact sur la santé des Québécois. De plus, la Loi sur les services de santé et les services sociaux reconnaît aux divers organismes de santé (CSSS, agences de santé) l'obligation de collaborer avec d'autres organismes de leur milieu afin d'améliorer la santé des Québécois. On constate donc que la mise en œuvre des politiques de santé s'articule dans dix-huit régions du Québec. Le ministère de la Santé et des Services sociaux doit établir les orientations pour ce qui a trait aux politiques sanitaires, mais la mise en œuvre de ces orientations relève des dix-huit agences qui doivent veiller à la mise en place des programmes et des actions sur leurs territoires. De même, ces dix-huit agences décentralisent l'application de ces programmes parmi les 
centres locaux de services communautaires (CLSC) de leurs territoires (quatre-vingt-quatorze en tout).

Le ministère des Transports déploie ses politiques au sein de quatorze directions territoriales, lesquelles doivent veiller à la mise en place des programmes. Ce sont ces directions, comme nous le précisions plus haut, qui travaillent en collaboration avec leurs partenaires régionaux afin de déterminer comment les ressources allouées à leur région seront affectées, en fonction des objectifs du ministère. Donc, non seulement les directions régionales ont la responsabilité de la mise en œuvre des politiques, alors que le ministère détermine les orientations générales, mais leurs actions doivent être concertées avec les partenaires de leur milieu, lesquels partenaires sont des entreprises privées ou communautaires qui exercent une influence dans le domaine des transports. Sont exclus de ces partenaires les organismes gouvernementaux, dont les agences de santé. Autrement dit, il n'y a pas d'évaluation spécifique ou externe. Les directions territoriales sont soumises aux règles de reddition de compte du ministère, comme toutes les directions du ministère. La figure 1 présente la formulation et la mise en œuvre au ministère de la Santé et des Services sociaux et au ministère des Transports du Québec.

\section{ILLUSTRATION : RÉGION DE MONTRÉAL}

L'Île-de-Montréal offre un exemple probant de mise en œuvre des politiques dans le domaine de la santé et des transports au Québec. Ainsi, le Plan de gestion des déplacements de Montréal (Québec, 2010) dresse une liste d'investissements de 2000 à 2010 qui atteignent 3838,27 millions de dollars canadiens (non actualisés). Pendant cette période, 1364,5 millions ont été dédiés aux autoroutes (parachèvements et améliorations), 115 millions sont allés à l'aménagement d'autoroutes au centre-ville, 2081 millions à divers pont et autoroutes et plus de 792 millions pour la réparation d'autoroutes existantes (Québec, 2010 : 68-71). Seuls 1556,22 millions de dollars ont été alloués aux transports en commun. Au total, ce sont plus de $74 \%$ des investissements du ministère des Transports dans la région de l'Île-de-Montréal qui ont été investis dans les transports non actifs de 2000 à 2010 (Québec, 2010, calculs des auteurs). De plus, le mot «santé » n'est mentionné à aucune reprise dans le Plan de gestion des déplacements, contrairement à ce qui est prévu dans les objectifs du Plan stratégique du ministère des Transports (Québec, 2008a) ainsi que dans la Politique québécoise du transport collectif (Québec, 2006b) et la Politique sur le vélo (Québec, 2008b).

Par ailleurs, l'Agence de la santé et des services sociaux de Montréal ne fait mention des transports sur son site Internet qu'à quelques reprises, contrairement à ce qui est prévu dans le Programme national de santé publique (Québec, 2008c) et dans le Plan d'action gouvernemental de promotion des saines habitudes de vie et de prévention des problèmes reliés au poids (Québec, 2006a). Par exemple, la section du site réservée aux publications de l'organisation compte 45 thématiques, dont une seule est liée aux transports : celle de la «Santé liée à l'environnement ». Dans celle-ci, plusieurs documents s'intéressent au bruit, à l'accès aux aliments santé, à la chaleur accablante, etc. D'autres documents font référence à l'environnement urbain (Paquin, Thérien, 2013a) et commentent des documents déjà approuvés par d'autres entités gouvernementales. En 2013, l'Agence a soumis un « [mémoire] au ministère des Transports du Québec dans le cadre de la consultation sur la Politique québécoise de mobilité durable »(Paquin, Thérien, 2013b). Ce mémoire visait à faire valoir la position de l'Agence de Montréal quant à l'aménagement du territoire et le transport collectif dans le cadre de la consultation menée par le ministère des Transports. Cet exemple illustre la densité de la hiérarchie dans le système québécois : l'Agence de Montréal défend l'un de ses objectifs en matière de mobilité non pas auprès de la direction territoriale des Transports, mais auprès du ministère des Transports. Le mémoire fut transmis au ministère et non à la direction territoriale parce que la direction territoriale n'a pas le mandat de recevoir ce mémoire. Seul le bureau attitré du ministère peut recevoir des mémoires; c'est le ministère qui organise les consultations publiques (et non pas les directions territoriales, lesquelles n'ont aucune autonomie juridique par rapport au ministère).

En somme, bien que les ministères des Transports et de la Santé et des Services sociaux du Québec formulent des objectifs cohérents les uns par rapport aux autres, il en va autrement dans leur mise en œuvre. Les organismes décentralisés sont respon- 
sables de cette dernière. Il en découle que le Plan de gestion des déplacements de Montréal, l'un des leviers importants du ministère des Transports dans cette région, ne mentionne ni ne semble tenir compte de la santé des Québécois ${ }^{1}$. Par ailleurs, l'Agence de la santé et des services sociaux de Montréal, l'organisme décentralisé du ministère de la Santé et des Services sociaux, fait mention à quelques reprises seulement de la santé en lien avec les transports sur son site Internet.

\section{Aveuglement des ACTeurs}

Il existe bien un décalage ou une incongruence entre la formulation et la mise en œuvre des politiques dans le domaine de la santé et dans le domaine des transports au Québec. Cette incongruence s'explique par une densité élevée de la hiérarchie. Cette densité élevée des hiérarchies est illustrée par le nombre élevé de pôles décisionnels, puisque dans le domaine de la santé chaque agence décide de la façon de mettre en œuvre les programmes et de la priorisation des objectifs des politiques en fonction des réalités régionales. Par exemple, l'Agence de la santé et des services sociaux de Montréal mentionne peu les transports sur son site Internet. De plus, les CLSC peuvent exercer des décisions similaires (mais à plus faible échelle); dans le domaine des transports, ce sont les directions régionales qui déterminent l'allocation des ressources et la priorisation des objectifs en fonction des besoins régionaux. Ceci concourt à créer un phénomène d'aveuglement des acteurs des politiques de santé et de transport. C'est-à-dire que, dans un contexte où les pôles décisionnels se multiplient verticalement à l'intérieur de plusieurs hiérarchies, les décideurs publics ne peuvent « voir» la façon dont les objectifs sont interprétés et priorisés, ainsi que la façon dont les programmes sont mis en œuvre sur l'ensemble du territoire ${ }^{2}$. Cet aveuglement des acteurs, que provoque l'incongruence de la mise en œuvre, crée une situation paradoxale : la formulation des politiques étant centralisée au sein

1. Il vaut la peine de souligner qu'au Québec, les lois ne prévoient pas de mise en œuvre; ce sont les énoncés de politique et les plans d'action des ministères qui prévoient la mise en œuvre. Ainsi, la mise en œuvre prévue dans le Plan de gestion des déplacements de Montréal n'a pas de valeur légale ou force de loi.

2. Au Québec, il y a peu d'évaluations par les ministères centraux des organismes décentralisés. Le système « central » a très peu d'influence sur la mise en œuvre. des ministères, on trouve une relative cohérence entre les politiques de santé et les politiques de transport; mais la mise en œuvre étant déconcentrée au sein de hiérarchies denses, la mise en œuvre de ces politiques est plutôt incohérente.

\section{Conclusion}

Cette note de recherche visait à déterminer si le gouvernement du Québec met de l'avant une approche cohérente dans un contexte où les enjeux relèvent de domaines de politiques différents (santé et transport). Nous avons fondé notre étude sur l'analyse de la cohérence des politiques publiques. Nous avons proposé de vérifier s'il existait un décalage entre la formulation et la mise en ouvre des politiques, qui s'expliquerait par une densité élevée de la hiérarchie et par la présence d'un grand nombre de pôles décisionnels.

Après avoir présenté les politiques du domaine de la santé et du domaine des transports du Québec, nous avons établi qu'il existe une mise en œuvre incongruente tant dans les politiques du domaine de la santé que dans les politiques du domaine du transport. Cette mise en œuvre incongruente s'explique par une densité assez élevée de la hiérarchie dans les deux domaines, la mise en œuvre étant décentralisée, dans un vaste réseau d'organisations régionales. Cette incongruence provoque un phénomène d'aveuglement des acteurs qui ouvre la voie à une priorisation et une interprétation différenciées des objectifs des politiques, ce qui, conséquemment, se solde en une pratique de mise en œuvre qui ne respecte pas les objectifs de la politique. L'effet de cette mise en œuvre incongruente est aussi paradoxal, puisque l'on se trouve devant une situation où la formulation des politiques de santé et de transport est cohérente, alors que la mise en œuvre de ces politiques est incohérente.

La centralisation de la formulation n'est pas, en soit, un facteur suffisant pour expliquer la cohérence des politiques, tout comme la décentralisation de la mise en œuvre au sein de denses hiérarchies ponctuées de grands nombres de pôles décisionnels n'est pas, non plus, une assurance d'une incohérence des politiques. Au contraire, des mécanismes, tels une collaboration obligatoire entre agences de Santé et directions territoriales des Transports, pourraient être mis de l'avant pour atténuer l'effet négatif sur 
la cohérence d'une telle décentralisation. Or, en l'absence de mécanismes de cohérence, il semble exister une incongruence de la mise en œuvre des politiques de santé et de transport québécoises.

\section{Bibliographie}

Bowen E.R., 1982. The Pressman-Wildavsky Paradox : Four Addenda or Why Models Based on Probability Theory can Predict Implementation Success and Suggest Useful Tactical Advice for Implementers, Journal of Public Policy, vol. 2, $n^{\circ} 1, p .1-21$.

Hupe P. L., 2010. The Thesis of Incongruent Implementation : Revisiting Pressman and Wildavsky, Public Policy and Administration, vol. 26, $\mathrm{n}^{\circ} 1$, p. 63-80.

Paquin S., Thérien F., (2013a). Développement urbain et santé. Mémoire sur le Plan de développement de Montréal, Agence de la santé et des services sociaux de Montréal, 15 p. [http:// publications.santemontreal.qc.ca/uploads/tx_asssmpublications/978-2-89673-310-1.pdf] Consulté le 7 septembre 2014.

Paquin S., Thérien F., 2013b. Une politique de mobilité durable: le point de vue de santé publique. Mémoire soumis au ministère des Transports du Québec dans le cadre de la consultation sur la Politique québécoise de mobilité durable, Agence de la santé et des services sociaux de Montréal, 15 p. [http://www.publications.santemontreal.qc.ca/uploads/ tx_asssmpublications/isbn978-2-89673-304-0.pdf] Consulté le 7 septembre 2014.

Pressman J. L., Wildavsky A.B., 1973. Implementation: How Great Expectations in Washington Are Dashed in Oakland. Or Why It's Amazing that Federal Programs Work At All. This Being a Saga of the Economic Development Administration as Told by Two Sympathetic Observers Who Seek to Build Morals on a Foundation of Ruined Hopes, Berkeley, University of California Press, 281 p.

Québec, 2006a. Plan d'action gouvernemental de promotion des saines habitudes de vie et de prévention des problèmes reliés au poids 2006-2012, 50 p. [http://msssa4.msss.gouv.qc.ca/frl document/publication.nsf/0/92885999c9ad58748525720d0 0653c6b?OpenDocument] Consulté le 7 septembre 2014.
Québec, 2006b. Politique québécoise du transport collectif, 59 p. [http://www.mtq.gouv.qc.ca/portal/page/portal/Librairiel Publications/fr/transport_collectif/compl_polit_collectif2006. pdf] Consulté le 7 septembre 2014.

Québec, 2008a. Plan stratégique 2008-2012, 30 p. [http://www. mtq.gouv.qc.ca/portal/page/portal/Librairie/Publications/fr/ ministere/plan_strat_2008_2012.pdf] Consulté le 7 septembre 2014.

Québec, 2008b. Politique sur le vélo, 28 p. [http://www.mtq.gouv. qc.ca/portal/page/portal/Librairie/Publications/fr/v\%E9lo/ velo_politique2008.pdf] Consulté le 7 septembre 2014.

Québec, 2008c. Programme national de santé publique 20032012 : mise à jour de 2008, 103 p. [http://publications.msss. gouv.qc.calacrobat/f/documentation/2008/08-216-01.pdf] Consulté le 7 septembre 2014.

Québec, 2010. Plan de gestion des déplacements région métropolitaine de Montréal, Pour une décongestion durable, 81 p. [http://www.mtq.gouv.qc.ca/portal/page/portal/ministerel ministere/plans_transport/montreal_plan_gestion_deplacements] Consulté le 7 septembre 2014.

Québec, 2013a. Plans de transport, [http://www.mtq.gouv. qc.ca/portal/page/portal/ministere/ministere/plans_transport] Consulté le 7 septembre 2014.

Québec, 2013b. Transports Québec - Organisation, [http://www. mtq.gouv.qc.ca/portal/page/portal/ministere/ministere/organisation] Consulté le 7 septembre 2014.

Québec, 2013c. Ministère des Transports du Québec [http://www. mtq.gouv.qc.ca/portal/page/portal/accueil] Consulté le 7 septembre 2014.

Québec, 2014a. Campagnes d'information, [http://www.msss. gouv.qc.ca/documentation/campagnes.php] Consulté le 7 septembre 2014 .

Québec, 2014b. Le système de santé et de services sociaux au Québec, 15 p. [http://publications.msss.gouv.qc.calacrobat/f/ documentation/2014/14-731-01F.pdf] Consulté le 7 septembre 2014.

Québec, 2014c. Santé environnementale, [http://www.msss. gouv.qc.ca/sujets/santepub/environnement/index.php?accueil] Consulté le 7 septembre 2014. 